\title{
Goal-Programming-Driven Genetic Algorithm Model for Wireless Access Point Deployment Optimization
}

\author{
Chen-Shu Wang ${ }^{1}$ and Ching-Ter Chang ${ }^{2}$ \\ ${ }^{1}$ Graduate Institute of Information and Logistics Management, National Taipei University of Technology, \\ Taipei 10608, Taiwan \\ ${ }^{2}$ Department of Information Management, Chang Gung University, Tao Yuan 333, Taiwan
}

Correspondence should be addressed to Chen-Shu Wang, wangcs@ntut.edu.tw

Received 24 February 2012; Accepted 8 May 2012

Academic Editor: Jung-Fa Tsai

Copyright (C 2012 C.-S. Wang and C.-T. Chang. This is an open access article distributed under the Creative Commons Attribution License, which permits unrestricted use, distribution, and reproduction in any medium, provided the original work is properly cited.

\begin{abstract}
Appropriate wireless access point deployment (APD) is essential for ensuring seamless user communication. Optimal APD enables good telecommunication quality, balanced capacity loading, and optimal deployment costs. APD is a typical NP-complex problem because improving wireless networking infrastructure has multiple objectives (MOs). This paper proposes a method that integrates a goal-programming-driven model (PM) and a genetic algorithm (GA) to resolve the MO-APD problem. The PM identifies the target deployment subject of four constraints: budget, coverage, capacity, and interference. The PM also calculates dynamic capacity requirements to replicate real wireless communication. Three experiments validate the feasibility of the PM. The results demonstrate the utility and stability of the proposed method. Decision makers can easily refer to the PM-identified target deployment before allocating APs.
\end{abstract}

\section{Introduction}

Appropriate wireless access point deployment (APD) is essential for ensuring seamless user communication. Optimal APD enables good telecommunication quality, balanced capacity loading, and optimal deployment costs. APD is a typical NP-complex problem [1] because it involves multiple decision objectives, such as budget [2-4], coverage [2,5-8], interference $[3,4,7]$, and dynamic capacity $[1,4,6-9]$. Furthermore, these objectives usually contradict each other [7]. For example, the number of APs is usually positively related to the wireless signal coverage rate and telecommunication reliability [1]. However, more APs increase deployment costs. These conflicting criteria should be considered simultaneously when solving APD problems $[9,10]$. 
Table 1: A comparison of the three wireless AP types.

\begin{tabular}{lcccc}
\hline AP Type & Cost (NT\$) & Range $(\mathrm{m})$ & Speed (Mbit/s) & Manufacturers \\
\hline $\begin{array}{l}\text { Type } 1 \\
\text { 802.11 b/g }\end{array}$ & 1365 & 30 to 89 & 54 & ASUS, D-Link, Lantech, SMC \\
$\begin{array}{l}\text { Type 2 } \\
\text { 802.11 b/g }\end{array}$ & 1710 & 33 to101 & 108 & Corega, D-Link, PCI, SMC \\
$\begin{array}{l}\text { Type 3 } \\
802.11 \mathrm{~b} / \mathrm{g} / \mathrm{n}\end{array}$ & 3138 & 51 to 163 & 300 & Apple, ASUS, Buffalo, Corega, D-Link \\
\hline
\end{tabular}

In the last decade, many studies have attempted to solve APD optimally by considering multiple objectives (MOs). There are four main objectives: budget, coverage rate, capacity, and interference. Studies have attempted to identify maximal coverage. For example, Huang et al. developed a growth-planning algorithm to establish the maximal coverage range [11]. Zhao et al. used a point-to-point signal strength strategy to implement indoor AP location optimization for maximal coverage [12]. For the capacity objective, the capacity requirements of wireless networks compared to wired networks are particularly difficult to evaluate because users are dynamic and can move from place to place. This makes APD a dynamic and complex problem. The dynamic capacity requirement must be addressed to resolve APD [13] because users can access particular APs to balance loads $[9,14]$. Finally, for the interference objective, too many APs of the same type and placed too close together may cause AP malfunction because of frequency interference. To avoid communication interference, some studies $[6,15]$ have suggested that APs should be arranged on different communication channels, but this leads to other communication channel assignment problems.

This paper applies a goal-programming-driven model (PM) to the MO-APD problem. It uses goal programming (GP) to infer and model the PM and a genetic algorithm (GA) to search for near optimal solutions. These methods are easily applied to MO-APD problems to reflect real situations. The remainder of this paper is organized as follows: Section 2 defines the problem; Section 3 details the PM; Section 4 presents a discussion on the PM solution process using a GA; Section 5 provides the results of numerical experiments which are given in this section; lastly, Section 6 offers a conclusion and suggestions for future research.

\section{Description of the APD Problem}

This research resolves the MO-APD problem according to four decision constraints: budget, coverage, capacity requirements, and interference. The PM identifies a feasible target deployment $(T)$, which consists of three types of wireless APs, as shown in Table 1 . This study conducted experiments and surveys that indicate that the coverage range and communication speed of a Type 3 AP are wider and faster, respectively, than AP Types 1 and 2. However, Type 1 AP equipment is cheaper than AP Types 2 and 3. Two APs may interfere with each other if they are the same type and are too near. Therefore, the PM must balance the four decision constraints and allocate three AP types in the target deployment of the APD problem. Table 2 lists the variables used in the proposed models. 
Table 2: Variables used in this paper.

\begin{tabular}{ll}
\hline Variable & Definition \\
\hline$B_{i j}$ & $\begin{array}{l}\text { The base station for the potential AP deployment area, where } i \text { is a row and } j \text { is a column } . i= \\
1,2, \ldots, n ; j=1,2, \ldots, m .\end{array}$ \\
$x_{q}$ & $\begin{array}{l}\text { Represents the type } q \text { AP allocated to } B_{i j}, \text { where } q \in[1,2,3] \text { in Table } 1 . \text { If } x_{q}=0 \text {, no AP has } \\
\text { been allocated to } B_{i j} .\end{array}$ \\
$c_{x_{q}}$ & $\begin{array}{l}\text { Represents the AP cost for each AP type, } x_{q} \in[1,2,3] . \\
\text { req }_{i j}\end{array} \quad$ The networking capacity requirement between $B_{i}$ and $B_{j}$. \\
\hline
\end{tabular}

\subsection{The Budget Constraint $(\theta(T))$}

Budget is the most important APD-MO constraint that directly affects the feasibility of $T$. The cost function in (2.1) evaluates the total cost of $T$. Equation (2.2) evaluates the budget constraint. In (2.2), bgt represents the given budget constraint for the AP allocation for $T$ :

$$
\begin{gathered}
\operatorname{CST}(T)=\sum_{i} \sum_{j} C_{x_{q}} B_{i, j}, \quad \forall X_{q} \neq 0, i=1,2, \ldots, n, j=1,2, \ldots, m, \\
\text { if } \operatorname{CST}(T)<\text { bgt, } \quad \theta(T)=1, \\
\text { else } \theta(T)=\frac{\text { bgt }}{\operatorname{CST}(T)} .
\end{gathered}
$$

\subsection{The Coverage Constraint $(\Phi(T))$}

Figure 1 shows that to enable seamless user communication, two APs are allocated, but two capacity requirements $\left(\mathrm{req}_{2,1}\right.$ and $\left.\mathrm{req}_{2,3}\right)$ have no signal coverage. The coverage function $(\mathrm{CVG}(T))$ evaluates the signal coverage area of $T$. Equation (2.3) evaluates the coverage fulfillment rate:

$$
\Phi(T)=\frac{\mathrm{CVG}(T)}{\text { target area }}
$$

\subsection{The Capacity Constraint $(\Psi(T))$}

Figure 2 shows a dynamic capacity scenario. The target area allocates two Type 1 APs $\left(\mathrm{AP}_{1}\right.$ and $\left.\mathrm{AP}_{2}\right)$, and two APs simultaneously cover the capacity requirements $\left(\mathrm{req}_{22}\right)$. For time slot $1\left(T_{1}\right)$, the capacity requirements of $\mathrm{req}_{12}, \mathrm{req}_{13}$, and req $\mathrm{q}_{22}$ access $\mathrm{AP}_{1}$, and the capacity requirements of req $q_{31}$ and $\mathrm{req}_{32}$ access $\mathrm{AP}_{2}$. Therefore, for time slot $T_{1}, \mathrm{AP}_{1}$ and $\mathrm{AP}_{2}$ must provide 55 and $25 \mathrm{mbit} / \mathrm{s}$ capacity, respectively. In time slot $T_{2}, \mathrm{req}_{22}$ shifts connection from $\mathrm{AP}_{1}$ to $\mathrm{AP}_{2}$ for balance loading. Therefore, for time slot $T_{2}, \mathrm{AP}_{1}$ and $\mathrm{AP}_{2}$ must provide 35 and $45 \mathrm{mbit} / \mathrm{s}$, respectively. Actual capacity requirements are difficult to evaluate accurately. 


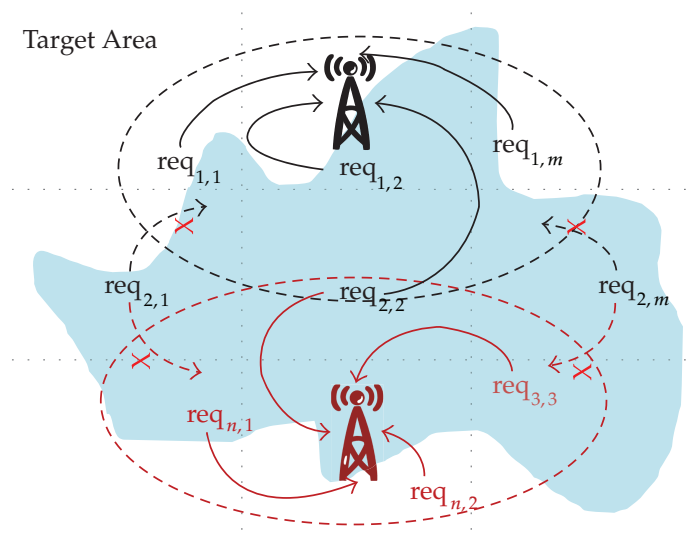

Figure 1: The signal coverage illustration.

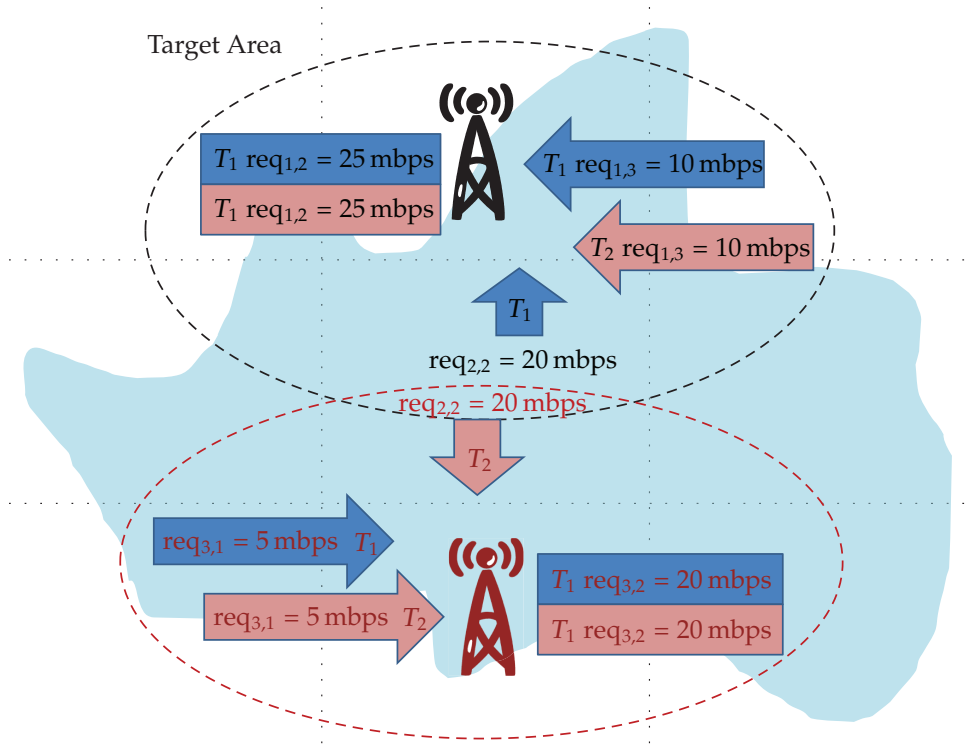

Figure 2: The dynamic capacity requirements of two APs. The blue area represents time slot $1\left(T_{1}\right)$ and the pink area represents time slot $2\left(T_{2}\right)$.

A Monte Carlo simulation algorithm-that simulates the capacity of $T$-implements the $\mathrm{DCP}_{i j}(T)$ function. Equation (2.4) evaluates the capacity fulfillment rate:

$$
\begin{aligned}
& \text { if } \operatorname{DCP}_{i j}(T)>\operatorname{req}_{i j}, \Psi(T)=1, i=1,2, \ldots, n ; j=1,2, \ldots, m, \\
& \text { else } \Psi(T)=\frac{\operatorname{DCP}_{i j}(T)}{\operatorname{req}_{i j}} .
\end{aligned}
$$




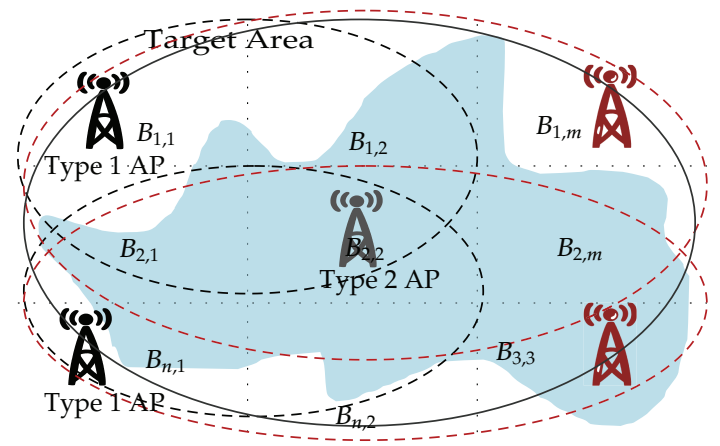

Figure 3: The frequency interference illustration.

\subsection{The Interference Constraint $(\omega(T))$}

Figure 3 shows that many Type 1 APs are used to maximize coverage and capacity of $T$ because of budget constraints. However, too many APs of the same type and allocated too near to each other may lead to AP malfunction because of AP frequency interference. For example, Figure 3 shows a perfect coverage design. However, it also shows an increased interference rate. The interference function (IFT(T)) evaluates the interference area of $T$. Equation (2.5) evaluates the interference fulfillment rate:

$$
\omega(T)=1-\frac{\operatorname{IFT}(T)}{\text { target area }} .
$$

\section{Proposed Model to Solve MO-APD}

Two main approaches can be used to formulate MO-APD. One approach is the cost-oriented approach, which aims to minimize total cost subject to MO performance constraints. This study formulated MO-APD using the cost-oriented approach as shown in Proposal 1 (P1). GAL-CVG, GAL-CP, and GAL-IFT are the given constraints for coverage rate, capacity fulfillment rate, and interference fulfillment rate, respectively.

(P1)

$$
\operatorname{Min} \operatorname{CST}(T) \leq \text { bgt, }
$$

subject to

$$
\begin{gathered}
\Phi(T) \geq \mathrm{GAL}-\mathrm{CVG}, \\
\Psi(T) \geq \mathrm{GAL}-\mathrm{CP}, \\
\omega(T) \geq \mathrm{GAL}-\mathrm{IFT} .
\end{gathered}
$$


Equations (3.2)-(3.4) are the coverage, capacity, and interference constraints. Equation (3.1) is the objective function, which minimizes the total cost subject to multiple decision constraints (3.2)-(3.4).

The second approach is performance oriented, and it maximizes the performance of target deployment subjects to real constraints (e.g., budget). This study reformulated the MOAPD using the performance-oriented method, shown in Proposal 2 (P2).

(P2)

$$
\operatorname{Max} \Phi(\mathrm{T})+\Psi(\mathrm{T})+\omega(\mathrm{T})
$$

subject to (3.1)-(3.4).

Equation (3.5) is the objective function in P2, which maximizes the coverage, capacity fulfillment, and interference fulfillment rates of $T$ subject to budgetary (3.1) and other decision constraints (3.2)-(3.4). GP aids MO decision-making problem modeling. It was first introduced by Charnes and Cooper [16] and further developed by Tamiz et al. [17], Romero [18], and Chang [19]. Various types of GP approaches exist, such as lexicographic GP, weighted GP, MINMAX (Chebyshev) GP, and multichoice GP [19]. To enable decision makers to easily set the constraint weighting according to their preferences, this study used a weighted GP approach to translate (P2) into the (PM). $w_{\mathrm{cvg}}, w_{\mathrm{cp}}$, and $w_{\mathrm{IFT}}$ are the important weights (between 0 and 1 ) for the GAL-CVG, GAL-CP, and GAL-IFT constraints, respectively.

$(\mathrm{PM})$

$$
\operatorname{Min} W_{\mathrm{bgt}}\left(\mathrm{bgt}^{+}\right)+W_{\mathrm{cvg}}\left(\mathrm{cvg}^{-}\right)+W_{\mathrm{cp}}\left(\mathrm{cp}^{-}\right)+W_{\mathrm{IFT}}\left(\mathrm{IFT}^{-}\right)
$$

subject to

$$
\begin{gathered}
\mathrm{CST}(T)-\mathrm{bgt}^{+}+\mathrm{bgt}^{-}=\mathrm{bgt}, \\
\Phi(T)-\mathrm{cvg}^{+}+\mathrm{cvg}^{-}=\mathrm{GAL}-\mathrm{CVG}, \\
\Psi(T)-\mathrm{cp}^{+}+\mathrm{cp}^{-}=\mathrm{GAL}-\mathrm{CP}, \\
\omega(T)-\mathrm{IFT}^{+}+\mathrm{IFT}^{-}=\mathrm{GAL}-\mathrm{IFT}, \\
\mathrm{bgt}^{+}, \mathrm{bgt}^{-}, \mathrm{cvg}^{+}, \mathrm{cvg}^{-}, \mathrm{cp}^{+}, \mathrm{cp}^{-}, \mathrm{IFT}^{+}, \mathrm{IFT}^{-} \geq 0
\end{gathered}
$$

\section{Process for Solving the PM Using a GA}

The GA is a stochastic searching method that uses the mechanics of natural selection to solve optimization problems. The GA was developed from the theory of natural selection [20]. Because the GA is a good stochastic technique for solving combinatorial optimization problems, this study uses the GA as the PM search tool, as shown in Figure 4.

An initial solution population is randomly created. The fitness of each individual in the population then determines whether it survives. Termination criteria (such as the generation size or the fitness value exceeding the threshold) determine the target deployment $(T)$ to be achieved. Finally, genetic operators such as selection, crossover, and mutation identify the 


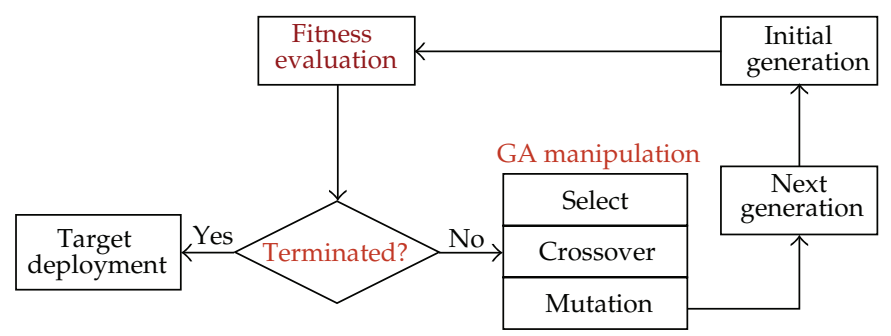

Figure 4: GA-based PM solution process.

next generation. After meeting a number of iterations or predefined criteria, a near optimal solution is found.

\subsection{Representation Structure: Encode/Decode}

A graph represents a target deployment $(T)$ that can also be expressed as a two-dimensional matrix. In the graph, each potential base station $\left(B_{i, j}\right)$ has two states: $\operatorname{AP}\left(B_{i, j}=1\right)$ allocated and no $\mathrm{AP}\left(B_{i, j}=0\right)$ allocated. A base station with an allocated AP must have an AP type $\left(x_{q}=[1,2,3]\right) . n \times m$ bit strings were used as chromosomes to represent $T$ :

$$
T=\left[\begin{array}{cccc}
B_{1,1} & B_{1,2} & \cdots & B_{1, m} \\
B_{2,1} & B_{2,2} & \cdots & B_{2, m} \\
\vdots & \vdots & \vdots & \vdots \\
B_{n, 1} & B_{n, 2} & \cdots & B_{n, m}
\end{array}\right] .
$$

\subsection{Evaluation Function}

The PM objective function $(F(i))$ was used as a GA evaluation function in (4.2). All variables in (4.2) are defined as in the PM:

$$
F(i)=W_{\mathrm{bgt}}\left(\mathrm{bgt}^{+}\right)+W_{\mathrm{cvg}}\left(\mathrm{cvg}^{-}\right)+W_{\mathrm{cp}}\left(\mathrm{cp}^{-}\right)+W_{\mathrm{IFT}}\left(\mathrm{IFT}^{-}\right)
$$

\subsection{GA Manipulations}

(1) Selection: roulette wheel selection ensures that highly fit chromosomes produce more offspring. This method selects a candidate network according to its survival probability, which is equal to its fitness relative to the whole population, as shown in (4.3):

$$
\left[\frac{F(i)}{\sum F(i)}\right]
$$

(2) Crossover: the crossover method randomly selects two chromosomes from the mating pool for mating. Crossover site $C$ is randomly selected in the interval 


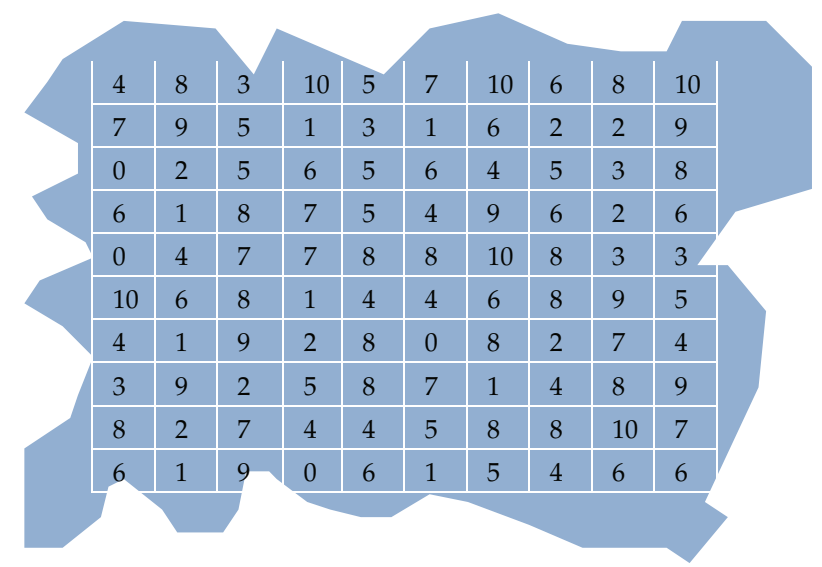

Figure 5: Capacity requirements for Experiment 1.

$[1, n \times m]$. Two new chromosomes, called offspring, are then obtained by swapping all characters between position $C$ and $n \times m$.

(3) Mutation: the combined reproduction and crossover methods occasionally lose potentially useful chromosome information. Mutation is introduced to overcome this. It is implemented by randomly complementing a bit (0 to 1 and vice versa). This ensures that good chromosomes are not permanently lost.

\section{Experiment Validation and Analysis}

To validate the efficiency and feasibility of the PM at resolving APD problems, three experiments were designed and implemented. Experiment 1 included four subtests to validate parameter combination types consisting of different decision variables. Experiment 2 included two subtests to confirm the ability of the PM to solve dynamic capacity problems. Experiment 3 ensured that the PM is suitable for large-scale problems and tested the GA parameter effects.

\subsection{Experiment 1: Decision Variable Combination Validation}

Four subtests consisting of different decision variables and important weights validated the ability of the PM to solve APD problems. The target area in Figure 5 is a $90 \mathrm{~km}^{2}$ irregularly shaped area. The capacity requirements in Figure 5 were identical for all subtests. All req $\mathrm{q}_{i j}$ could move around the target area, where signal coverage was present. For comparative purposes, the GA parameters of the four subtests-population size (600), terminated generation (500), crossover rate (0.4), and mutation rate (0.1) —were fixed. Table 3 lists the other decision variables.

Table 4 shows the four subtests formulated as Model I and Model II according to the PM. To avoid the randomizing effect of the GA, all subtests were run three times with the same parameters on the same machine. The result averages are reported. Table 5 shows the analysis of the experiment results. The E1.a and E1.b results show that the important budget weight is less in E1.b than in E1.a. Therefore, only 15 APs (on average) are deployed for E1.b, 
Table 3: Decision variables and import weights for four subtests in Experiment 1.

\begin{tabular}{|c|c|c|c|}
\hline Decision variable & E1.a & E1.b & E1.d \\
\hline Budget & $30000\left(W_{\text {bgt }}=.25\right)$ & $30000\left(W_{\text {bgt }}=.40\right)$ & $35000\left(W_{\text {bgt }}=.25\right)$ \\
\hline Coverage rate & $85 \%\left(W_{\mathrm{cvg}}=.25\right)$ & $85 \%\left(W_{\mathrm{cvg}}=.20\right)$ & $85 \%\left(W_{\mathrm{cvg}}=.25\right)$ \\
\hline Capacity fulfillment rate & $80 \%\left(W_{\mathrm{cp}}=.25\right)$ & $80 \%\left(W_{\mathrm{cp}}=.20\right)$ & $80 \%\left(W_{\mathrm{cp}}=.25\right)$ \\
\hline Interference fulfillment rate & $80 \%\left(W_{\mathrm{IFT}}=25\right)$ & $80 \%\left(W_{\mathrm{IFT}}=.20\right)$ & $80 \%\left(W_{\mathrm{IFT}}=25\right) \quad 90 \%\left(W_{\mathrm{IFT}}=25\right)$ \\
\hline
\end{tabular}

Note that important weights are marked in parentheses.

Table 4: Model I and Model II for resolving the four subtests.

\begin{tabular}{ll}
\hline Model I (for subtests E1.a and E1.b) & Model II (for subtests E1.c and E1.d) \\
\hline Min 0.25 $\mathrm{a}(0.4)^{\mathrm{b}} \mathrm{bgt}^{+}+0.25^{\mathrm{a}}(0.2)^{\mathrm{b}} \mathrm{cvg}^{-}+$ & Min 0.25bgt ${ }^{+}+0.250 \mathrm{cvg}^{-}+0.25 \mathrm{cp}^{-}+0.25 \mathrm{IFT}^{-}$ \\
$0.25^{\mathrm{a}}(0.2)^{\mathrm{b}} \mathrm{cp}^{-}+0.25^{\mathrm{a}}(0.2)^{\mathrm{b}} \mathrm{IFT}^{-}$ & Subject to \\
Subject to & $\mathrm{CST}(T)-\mathrm{bgt}^{+}+\mathrm{bgt}^{-}=30000$ \\
$\mathrm{CST}(T)-\mathrm{bgt}^{+}+\mathrm{bgt}^{-}=30000$ & $\Phi(T)-\mathrm{cvg}^{+}+\mathrm{cvg}^{-}=0.85$ \\
$\Phi(T)-\mathrm{cvg}^{+}+\mathrm{cvg}^{-}=0.85$ & $\Psi(T)-\mathrm{cp}^{+}+\mathrm{cp}^{-}=0.8$ \\
$\Psi(T)-\mathrm{cp}^{+}+\mathrm{cp}^{-}=0.8$ & $\omega(T)-\mathrm{IFT}^{+}+\mathrm{IFT}^{-}=0.8^{\mathrm{c}}(0.9)^{\mathrm{d}}$ \\
$\omega(T)-\mathrm{IFT}^{+}+\mathrm{IFT}^{-}=0.8$ & $\mathrm{bgt}^{+}, \mathrm{bgt}^{-}, \mathrm{cvg}^{+}, \mathrm{cvg}^{-}, \mathrm{cp}^{+}, \mathrm{cP}^{-}, \mathrm{IFT}^{+}, \mathrm{IFT}^{-} \geq 0$ \\
\hline
\end{tabular}

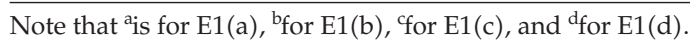

Table 5: Analysis of the results of Experiment 1.

\begin{tabular}{lcccc}
\hline Indicator & E1.a & E1.b & E1.c & E1.d \\
\hline Fitness & .9534 & .9420 & .9568 & .9567 \\
Time $(\mathrm{s})$ & 160.0993 & 160.106 & 159.0593 & 170.4233 \\
Cost & 36954 & 29360 & 34647 & 34694 \\
Coverage rate & .85 & .6967 & .8167 & .8233 \\
Capacity fulfillment rate & .8490 & .6981 & .8189 & .8269 \\
Interference fulfillment rate & .94 & .9867 & .94 & .99 \\
Number of APs & 22 & 15 & 21 & 23 \\
\hline
\end{tabular}

as shown in Figure 6. The results also show that the decision maker must either increase the budget or adjust the other decision objectives. For example, E1.b shows that the coverage and capacity fulfillment rates can only reach 0.7 at the current budget. As the budget increases from 30000 (in E1.b) to 35000 (in E1.c and E1.d), the number of APs deployed increases to 22 (on average). The coverage and capacity rates increase from 0.7 in E1.b to 0.82 in E1.c and E1.d. E1.d deployed more APs (23) at a lower cost than E1.a (22 APs). Figure 6 shows that the APs in E1.c and E1.d are spread evenly in the target area to avoid interference. Figure 7 shows the convergence trends for all subtests. $T$ emerges after 100-150 iterations.

\subsection{Experiment 2: Dynamic Capacity Requirement Validation}

Experiment 2 consisted of two subtests to validate the ability of the PM to resolve dynamic capacity requirements. Figure 6(a) shows that in subtest E2.a, most capacity requirements are in the central area of the target $\left(32 \mathrm{~km}^{2}\right)$. Figure $6(\mathrm{~b})$ shows that in subtest E2.b, the capacity 


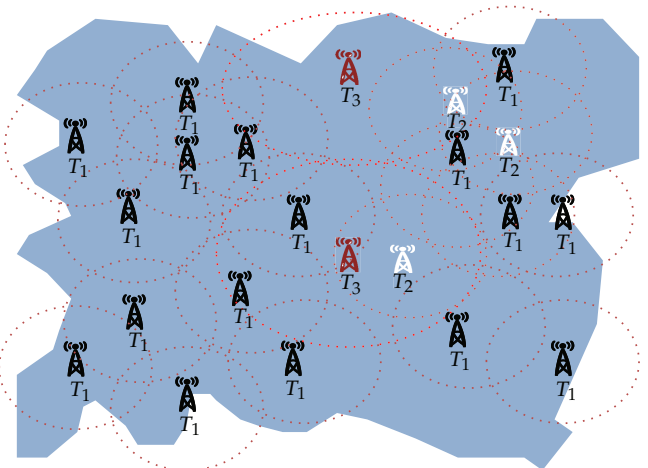

E1.a

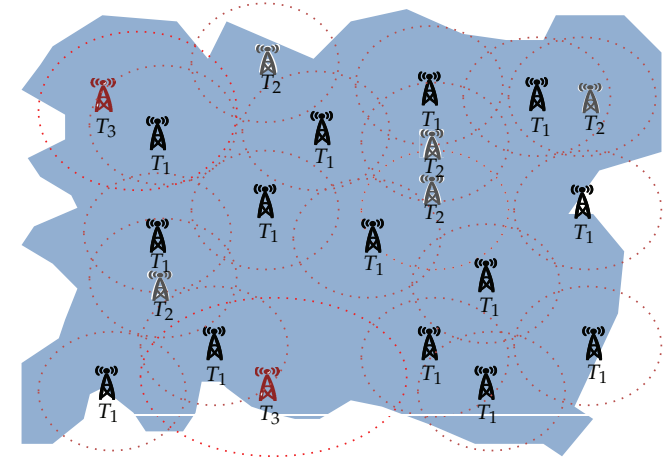

E1.c

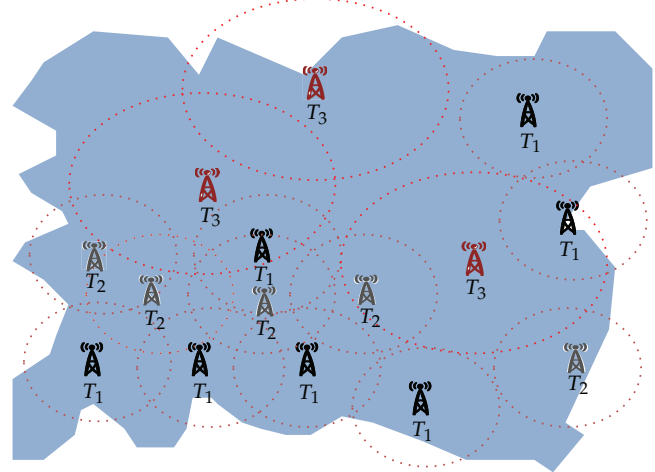

E1.b

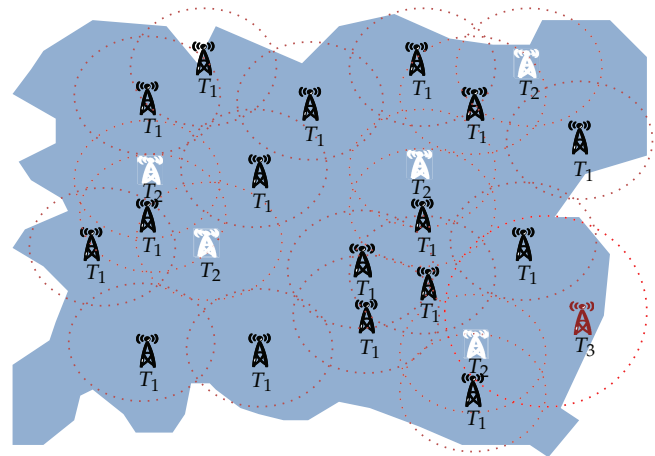

E1.d

Figure 6: AP deployment results for the four subtests in Experiment 1.

Table 6: Decision variable and important weights for Experiment 2.

\begin{tabular}{lc}
\hline Decision variable & Variable value \\
\hline Budget & $15000\left(W_{\text {bgt }}=.3\right)$ \\
Coverage rate & $85 \%\left(W_{\text {cvg }}=.15\right)$ \\
Interference fulfill rate & $85 \%\left(W_{\text {IFT }}=.15\right)$ \\
Capacity fulfill rate & $95 \%\left(W_{\mathrm{cp}}=.4\right)$ \\
\hline
\end{tabular}

Note that the important weights are marked in parentheses.

requirements are scattered in the corners of the target area. Table 6 shows that the capacity requirements and all default decision variables are identical for both tests. The GA parameters-population size (600), terminated generation (500), crossover rate (0.4), and mutation rate (0.1) -were fixed to enable result comparison. To avoid random GA effects, all subtests were run three times with the same parameters on the same machine. The result averages are reported.

Table 7 shows the experiment results analysis. As expected, APD follows the capacity requirements, as shown in Figures 8(a) and 8(b). Figures 8(a) and 8(b) also show that APs are more central in E2.a than in E2.b to fulfill the capacity requirements. Although the capacity requirements are the same in both experiments, E2.a requires nine APs, which is more than 


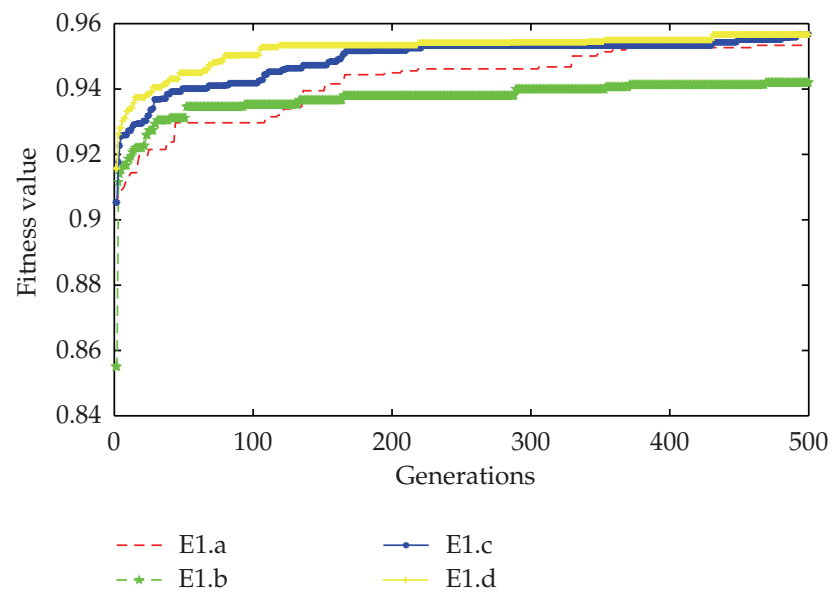

Figure 7: The convergence trends for the four subtests in Experiment 1.

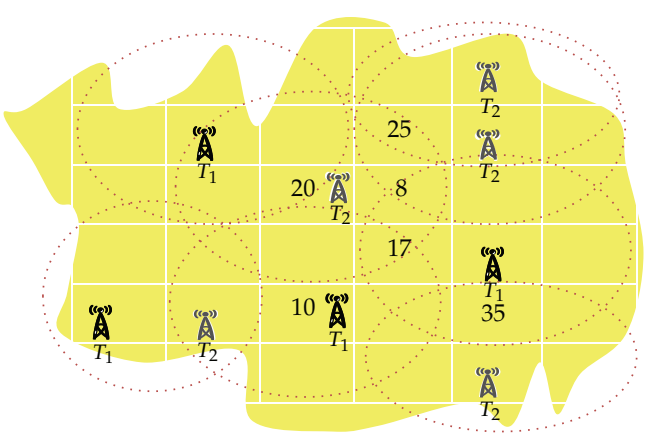

(a)

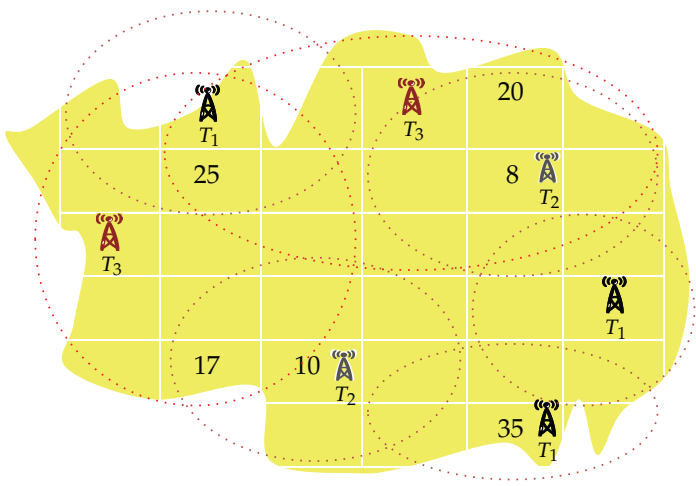

(b)

Figure 8: The capacity requirement and results of Experiment 2 for (a) subtest E2.a and (b) subtest E2.b. The numbers represent the capacity requirements.

E2.b (seven APs). Therefore, capacity requirements are dynamic, and $T$ requires more APs to manage the capacity requirement increase in E2.a.

\subsection{Experiment 3: The Effect of Large-Scale Problems and GA Parameters on Validation}

Two subtests of Experiment 3 were designed as large-scale problems. Table 8 lists the decision variables and the important weights. Experiment 3 also tested GA parameter combinations, including crossover and mutation rates.

The results analysis in Table 9 shows that the PM is more sensitive to crossover rate. Generation - as an evaluation indicator for subtests E3.a and E3.b-shows that feasible deployment can be reached in the following order of crossover rates: 0.6 (converged by 22 iterations), 0.4 (converged by 70 iterations), and 0.2 (converged by 95 iterations). However, no such pattern exists for mutation rate in either test. Therefore, a crossover rate of 0.4 and 
Table 7: Analysis of the results of Experiment 2.

\begin{tabular}{lcc}
\hline Indicator & E2.a & E2.b \\
\hline Fitness & 1 & 1 \\
Time (s) & 5.1883 & 4.7797 \\
Cost & 14387 & 14068 \\
Coverage rate & .8518 & .8518 \\
Capacity fulfillment rate & 1 & 1 \\
Interference fulfillment rate & .9815 & .9259 \\
Number of APs & 9 APs & 7 APs \\
\hline
\end{tabular}

Table 8: Decision variables and important weights for Experiment 3.

\begin{tabular}{lcc}
\hline Objective & E3.a & E3.b \\
\hline Target area & $2250 \mathrm{~km}^{2}$ & $90000 \mathrm{~km}^{2}$ \\
Budget $\left(W_{\mathrm{bgt}}=.55\right)$ & 1350000 & 5650000 \\
Coverage rate $\left(W_{\mathrm{cvg}}=.15\right)$ & $85 \%$ & $85 \%$ \\
Capacity and interference fulfillment rates $\left(W_{\mathrm{cp}}=W_{\mathrm{IFT}}=0.15\right)$ & $80 \%$ & $80 \%$ \\
\hline
\end{tabular}

Note that the capacity requirements are shown in the Appendix and the important weights are marked in parentheses.

Table 9: Analysis of the results of Experiment 3.

\begin{tabular}{lccccc}
\hline \multirow{2}{*}{ Indicator } & \multicolumn{2}{c}{ Fixed mutation rate $(m=0.1)$} & \multicolumn{2}{c}{ Fixed crossover rate $(c=0.4)$} \\
& $C=0.2$ & $C=0.4$ & $C=0.6$ & $m=0.01$ & $M=0.2$ \\
\hline Fitness & $1(.9869)$ & $1(1)$ & $1(1)$ & $1(1)$ & $1(1)$ \\
Generation & $96(93)$ & $53(86)$ & $18(26)$ & $9(94)$ & $22(62)$ \\
Time & 489.2550 & 526.2310 & 552.5640 & 496.0540 & 541.5390 \\
& $(3795.2)$ & $(5080.5)$ & $(3880.6)$ & $(3334.8)$ & $(4866.5)$ \\
Cost & $1,349,670$ & $1,326,549$ & $1,347,933$ & $1,348,896$ & $1,324,479$ \\
& $(5,736,480)$ & $(5,593,797)$ & $(5,576,205)$ & $(5,580,897)$ & $(5,566,053)$ \\
Capacity fulfillment rate & .8485 & .8384 & .8495 & .8450 & .8531 \\
& $(.8641)$ & $(.8536)$ & $(.8486)$ & $(.8502)$ & $(.8560)$ \\
Coverage fulfillment rate & .8500 & .8496 & .8484 & .8496 & .8496 \\
& $(.8536)$ & $(.8634)$ & $(.8506)$ & $(.8510)$ & $(.8559)$ \\
Interference & .8996 & .9100 & .8996 & .9048 & .9012 \\
& $(.8929)$ & $(.8914)$ & $(.8891)$ & $(.8987)$ & $(.8952)$ \\
\hline
\end{tabular}

The experiment results for E3(b) are in parentheses.

a mutation rate of 0.1 are recommended for experiment implementation to avoid GA parameter effects. The results in Table 9 show that large-scale problems (E3.b) can be resolved within an acceptable time (4191.52 s, approximately $1 \mathrm{~h}$ ).

\section{Conclusion}

Optimal wireless LAN (WLAN) design is important to ensure seamless user communication. Appropriately locating wireless APs for WLANs is important. Optimal APD enables high telecommunication quality, balanced capacity loading, and optimal deployment costs. This study proposes a GP-driven model integrated with a GA to solve MO-APD subject to four constraints: budget, capacity, interference, and coverage. The experiment results show that 


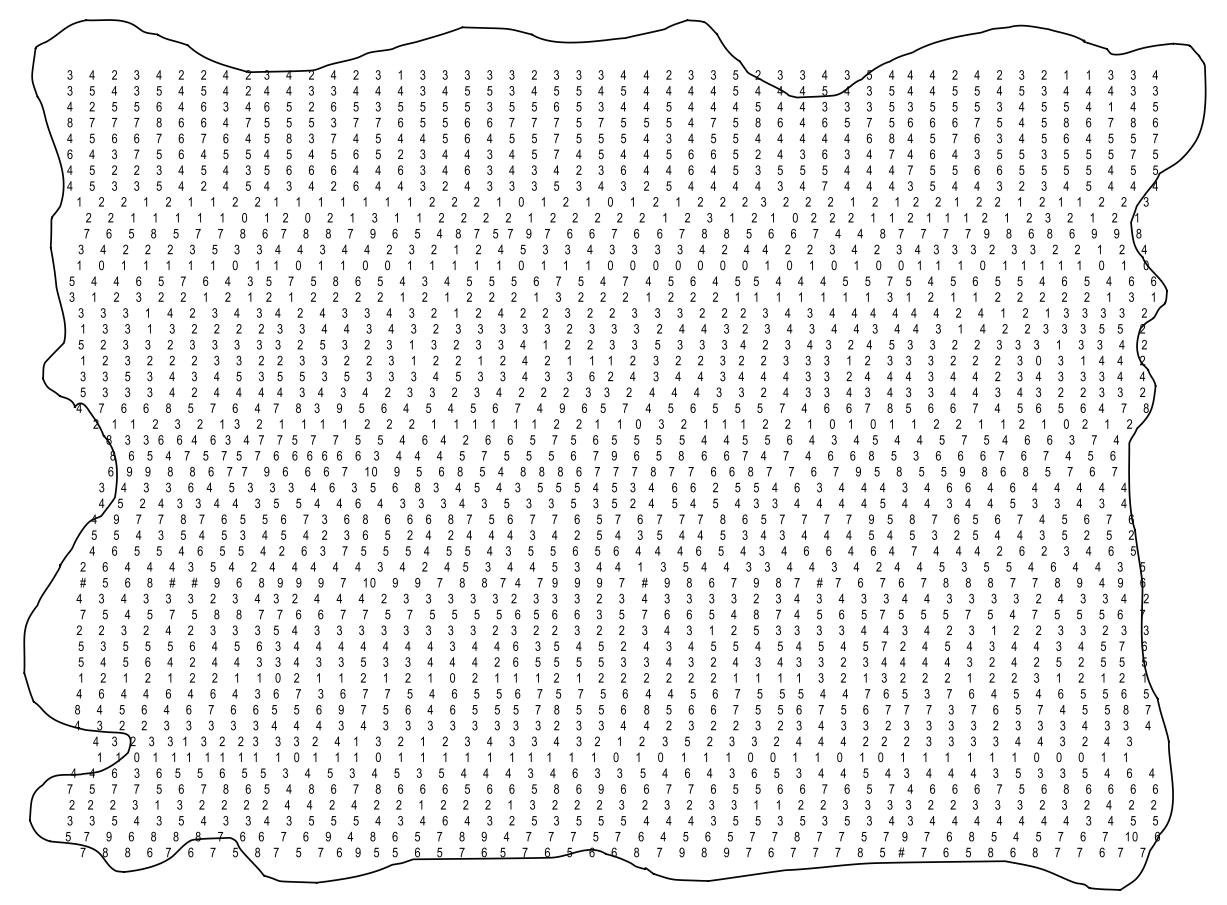

Figure 9: Capacity requirements for Experiment 3.a.

the PM resolves many APD problems and achieves dynamic capacity replication. Results confirm the ability of the PM to solve large-scale APD problems. Future research should focus on other applications and further verification of PM.

\section{Appendix}

Figure 9 shows the capacity requirement for the E3.a subtest.

\section{References}

[1] N. Weicker, G. Szabo, K. Weicker, and P. Widmayer, "Evolutionary multiobjective optimization for base station transmitter placement with frequency assignment," IEEE Transactions on Evolutionary Computation, vol. 7, no. 2, pp. 189-203, 2003.

[2] A. Mc Gibney, M. Klepal, and D. Pesch, "Agent-based optimization for large scale WLAN design," IEEE Transactions on Evolutionary Computation, vol. 15, no. 4, pp. 470-486, 2011.

[3] L. Liao, W. Chen, C. Zhang, L. Zhang, D. Xuan, and W. Jia, "Two birds with one stone: wireless access point deployment for both coverage and localization," IEEE Transactions on Vehicular Technology, vol. 60, no. 5, pp. 2239-2252, 2011.

[4] K. P. Scheibe and C. T. Ragsdale, "A model for the capacitated, hop-constrained, per-packet wireless mesh network design problem," European Journal of Operational Research, vol. 197, no. 2, pp. 773-784, 2009.

[5] K. Collins, S. Mangold, and G. M. Muntean, "Supporting mobile devices with wireless LAN/MAN in large controlled environments," IEEE Communications Magazine, vol. 48, no. 12, pp. 36-43, 2010.

[6] A. Hills, "Large-scale wireless LAN design," IEEE Communications Magazine, vol. 39, no. 11, pp. 98$104,2001$. 
[7] K. Jaffrès-Runser, J. M. Gorce, and S. Ubéda, "Mono- and multiobjective formulations for the indoor wireless LAN planning problem," Computers and Operations Research, vol. 35, no. 12, pp. 3885-3901, 2008.

[8] R. Whitaker and S. Hurely, "Evolution of planning for wireless communication systems," in Proceedings of the 36th Hawaii International Conference on System Sciences, p. 10, January 2003.

[9] J. H. Lee, B. J. Han, H. J. Lim, Y. D. Kim, N. Saxena, and T. M. Chung, "Optimizing access point allocation using genetic algorithmic approach for smart home environments," Computer Journal, vol. 52, no. 8, pp. 938-949, 2009.

[10] I. E. Liao and K. F. Kao, "Enhancing the accuracy of WLAN-based location determination systems using predicted orientation information," Information Sciences, vol. 178, no. 4, pp. 1049-1068, 2008.

[11] X. Huang, U. Behr, and W. Wiesbecd, "Automatic cell planning for a low-cost and spectrum efficient wireless network," in Proceedings of the IEEE Global Telecommunications Conference (GLOBECOM '00), vol. 1, pp. 282-276, December 2000.

[12] Y. Zhao, H. Zhou, and M. Li, "Indoor access points location optimization using Differential Evolution," in Proceedings of the International Conference on Computer Science and Software Engineering (CSSE '08), pp. 382-385, December 2008.

[13] J. H. Lee, B. J. Han, H. K. Bang, and T. M. Chung, “An optimal access points allocation scheme based on genetic algorithm," in Proceedings of the International Conference on Future Generation Communication and Networking (FGCN'07), pp. 55-59, December 2007.

[14] F. Guo and T. C. Chiueh, "Scalable and robust WLAN connectivity using access point array," in Proceedings of the International Conference on Dependable Systems and Networks, pp. 288-297, July 2005.

[15] X. Ling and K. L. Yeung, "Joint access point placement and channel assignment for 802.11 wireless LANs," IEEE Transactions on Wireless Communications, vol. 5, no. 10, pp. 2705-2711, 2006.

[16] A. Charnes and W. W. Cooper, Management Model and Industrial Application of Linear Programming, vol. 1, Wiley, New York, NY, USA, 1961.

[17] M. Tamiz, D. Jones, and C. Romero, "Goal programming for decision making: an overview of the current state-of-the-art," European Journal of Operational Research, vol. 111, no. 3, pp. 569-581, 1998.

[18] C. Romero, "Extended lexicographic goal programming: a unifying approach," Omega, vol. 29, no. 1, pp. 63-71, 2001.

[19] C. T. Chang, "Revised multi-choice goal programming," Applied Mathematical Modelling, vol. 32, no. 12, pp. 2587-2595, 2008.

[20] D. E. Goldberg, Genetic Algorithms in Search, Optimization and Machine Learning, Addison-Wesley, 1989. 


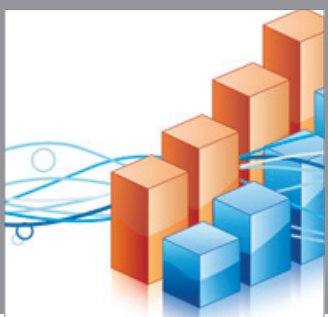

Advances in

Operations Research

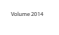

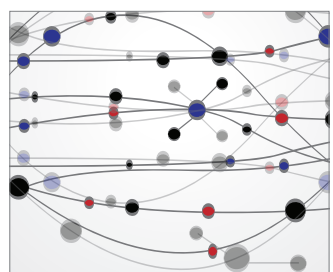

\section{The Scientific} World Journal
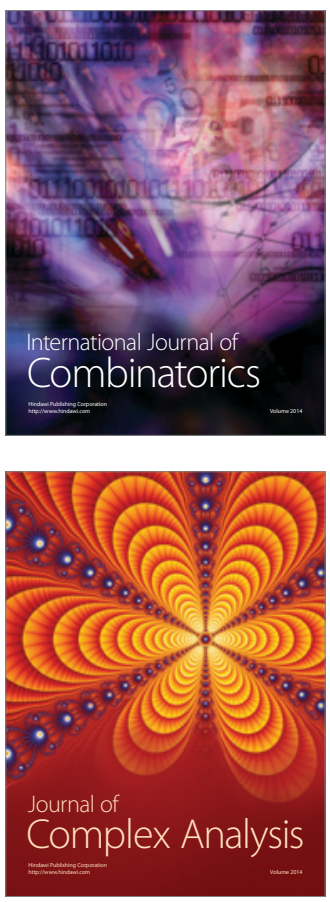

International Journal of

Mathematics and

Mathematical

Sciences
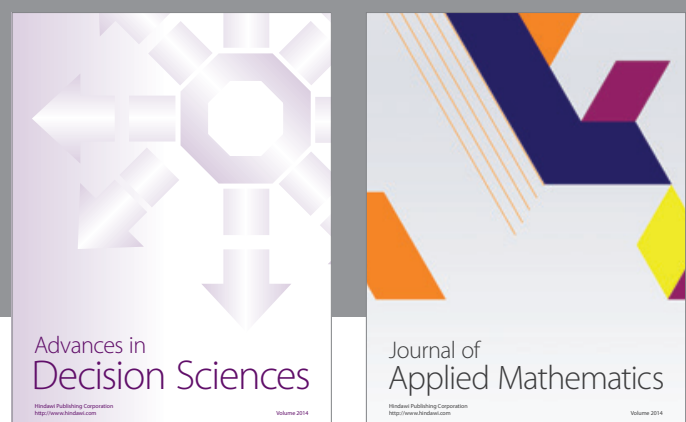

Journal of

Applied Mathematics
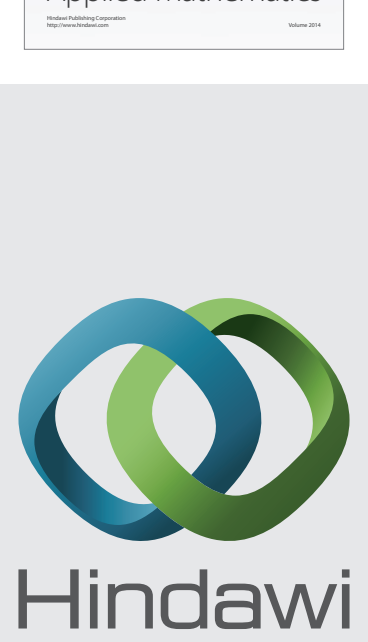

Submit your manuscripts at http://www.hindawi.com
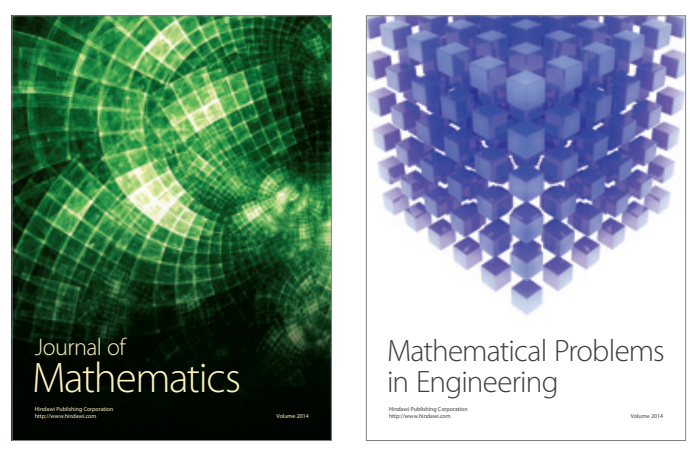

Mathematical Problems in Engineering
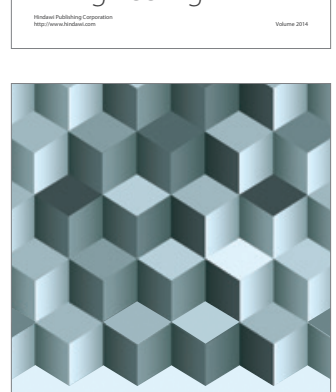

Journal of

Function Spaces
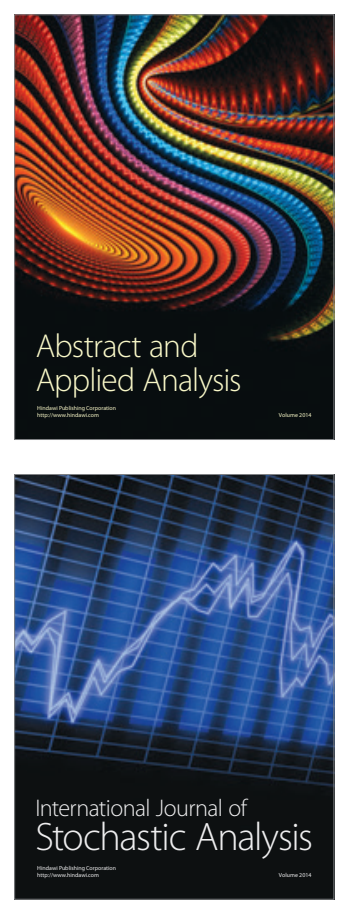

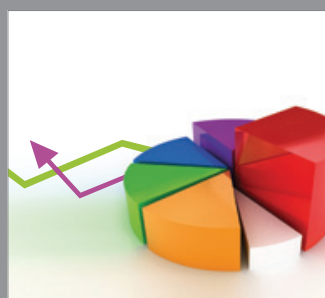

ournal of

Probability and Statistics

Promensencen
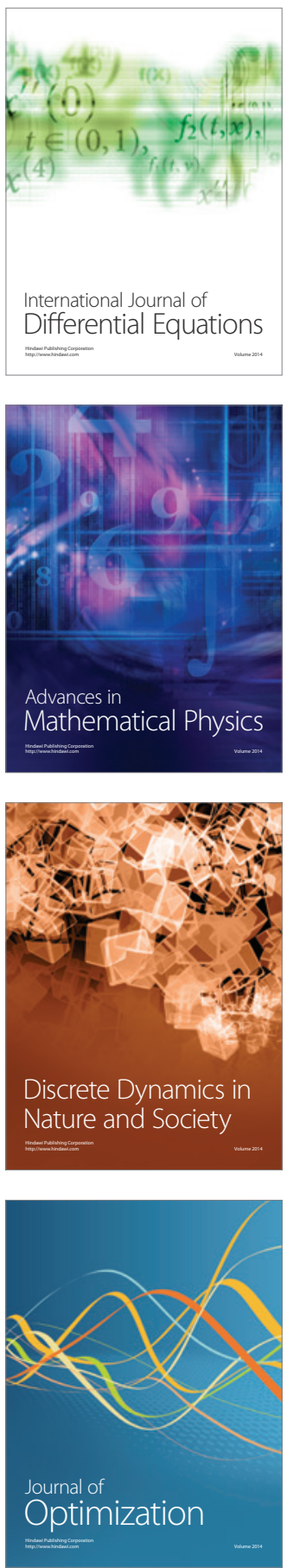